\title{
Airway and vascular maturation stimulated by tracheal occlusion do not correlate in the rabbit model of diaphragmatic hernia
}

\author{
Jordi Prat Ortells ${ }^{1}$, Asteria Albert' ${ }^{1}$, Xavier Tarrado', Lucas Krauel', Rogelio Cruz ${ }^{2}$, Óscar Moreno-Álvarez ${ }^{2}$, Victoria Fuste ${ }^{3}$ and \\ Montserrat Castañón'
}

BACKGROUND: In animal models of congenital diaphragmatic hernia (CDH), tracheal occlusion (TO) has induced maturation of both airway spaces and vascular structures. Airway and vascular response to TO are assumed to occur in parallel. This study aims to describe and measure the relationship between airway and vascular maturation induced by TO.

METHODS: A rabbit model of $\mathrm{CDH}$ on gestational day (GD) 23 and TO on GD 28 (term = GD 31) has been used. Two study groups have been defined: $\mathrm{DH}$ (diaphragmatic hernia) and TO (DH treated with TO). Animals were collected on GD 30 and blood flow data of the pulmonary artery (pulsatility index (PI) and fractional moving blood volume) were ultrasonographically measured. Lung morphometry consisted of measurements of radial alveolar count (RAC) and arterial muscular thickness.

RESULTS: Animals in the DH group $(n=9)$ had the worst hemodynamic parameters; their lungs were hypoplastic and had the thickest arterial muscular layer. Animals in the TO group $(n=10)$ had all these effects reversed. There were no correlations among hemodynamic, airway, and vascular parameters, except for RAC and PI ( $r=-0.528, P=0.043)$.

CONCLUSION: Airway and vascular maturation after TO appear to be uncorrelated effects. TO could trigger several pathways that separately regulate airway and vascular responses.

C ongenital diaphragmatic hernia $(\mathrm{CDH})$ is a diaphragmatic defect associated with lung hypoplasia, affecting both the airway spaces and the lung vasculature (1). This is so because the lung vascular tree development parallels that of lung parenchyma, closely following the bronchial branching. In case of $\mathrm{CDH}$, pulmonary development is impaired at an early stage, usually at the beginning of the pseudoglandular phaseat about 10 gestational wk-preventing further bronchial budding and branching $(1,2)$. Alveolization will be proportionally reduced. This is the cause of pulmonary hypoplasia associated with $\mathrm{CDH}$. In severe cases, there are just not enough effective pulmonary units to sustain life. The pulmonary arterial tree is also reduced. Structural changes are profound: arteries retain their thick muscular layer distally, as far as the intra-acinar bronchi; the capillary network surrounding the alveoli is poor and thick walled; arteries have a higher muscular tone and sensitivity to hypoxia (3). This is the pathophysiological basis for the persistent pulmonary hypertension that complicates the management of patients with $\mathrm{CDH}$. Consequently, the two main causes of neonatal death in $\mathrm{CDH}$ are persistent pulmonary hypertension and pulmonary hypoplasia (4-6). If a high risk for these conditions is prenatally identified, fetal treatment could provide the only means to improve postnatal outcome. Presently, tracheal occlusion (TO) is the only accepted fetal treatment for $\mathrm{CDH}$, and it is offered in a few selected centers.

TO promotes lung airway maturation that leads to an increase in airway budding and total functional alveolar units (7-9). Among CDH fetuses that are at the verge of viability, treatment with TO may reduce pulmonary hypoplasia and increase their chance of survival.

In addition, TO promotes vascular growth and development, reducing the thickness of the muscular layer in pulmonary arteries in sheep, rabbit, and rat models of DH (10-15). Our group demonstrated hemodynamic improvement in rabbit fetuses with DH following TO (16). The vascular effect of TO is to reduce pulmonary hypertension, thus placing the patient in a better condition for postnatal management.

Fetuses that achieve a significant pulmonary growth after TO are expected to demonstrate greater vascular and hemodynamic stability. However, in a clinical context, this is not always the case. Indeed, a correlation of the hemodynamic, vascular, and morphological effects promoted by TO has not been assessed. Closing this gap is the aim of this study.

\section{RESULTS}

Forty pregnant rabbits were operated on. There were two deaths due to respiratory depression during anesthesia. Diaphragmatic hernia was attempted in 133 fetuses. After

\footnotetext{
'Department of Pediatric Surgery, Hospital Sant Joan de Déu-Hospital Clínic, Universitat de Barcelona, Barcelona, Spain; ${ }^{2}$ Fetal and Perinatal Medicine Research Group, Department of Maternal-Fetal Medicine, Hospital Clinic-IDIBAPS, Universitat de Barcelona and Centre for Biomedical Research on Rare Diseases (CIBER-ER), Barcelona, Spain; ${ }^{3}$ Department of Pathology, Hospital Clínic, Universitat de Barcelona, Barcelona, Spain. Correspondence: Jordi Prat Ortells (joprat@hsjddbcn.org) 
the first 50 unsuccessful attempts, 36 out of $83 \mathrm{DH}$ (a rabbit model of $\mathrm{CDH}$ ) fetuses survived. Seventeen of them underwent TO. Twenty-five subjects finished the experiment, of which 19 were suitable for this study. Six fetuses were excluded for different reasons: in three cases, all the airway spaces were filled by leukocytes, making morphometry impossible; one TO ( $\mathrm{DH}$ animals treated later with $\mathrm{TO}$ ) fetus did not have DH; in one DH fetus, there was no visceral herniation into the chest; and one more fetus was excluded due to a mistake in the labeling of the samples. The DH group consisted of 9 subjects with DH; the TO group consisted of 10 subjects with $\mathrm{DH}$ and TO treatment; 17 unoperated littermates were also collected.

\section{Hemodynamics}

Pulsatility index (PI) of DH group was higher than that of TO group $(11.4 \pm 3.8$ vs. $5.5 \pm 2.2 ; P<0.05)$. Fractional moving blood volume (FMBV) was larger in TO group than in $\mathrm{DH}$ group (TO: $28 \pm 7.2$ vs. $\mathrm{DH}: 15.6 \pm 7.3 \% ; P<0.05$ ). Neither the peak of early diastolic reversed flow nor peak of maximum systolic speed flow showed significant differences between the groups.

\section{Morphological Study}

Both airway estimators of pulmonary hypoplasia, lung-tobody weight ratio (LBWR) and radial alveolar count (RAC), were significantly lower in $\mathrm{DH}$ group (LBWR: $1.27 \pm 0.38$; RAC: $2.8 \pm 0.4$ ) than in TO group (LBWR: $1.96 \pm 0.81$; RAC: $3.9 \pm 0.34)$. Moreover, RAC in TO group did reach that of unoperated littermates $(4.7 \pm 1.0, P=0.80)$.

\section{Vascular Study}

The results of arterial muscular thickness (AMT) are shown in Figure 1. The arteries have been distributed in size groups to reveal any differences in muscularization related to the branching order. Four hundred and twenty-five arterial measurements have been obtained in DH group and 484 in TO group, that is, all the arteries identified in the selected lung section of each subject. Eight hundred and twelve arteries of normal individuals have also been measured. AMT shows a peak in the 15 - to $30-\mu \mathrm{m}$ diameter range in both groups, as well as in normal siblings. For any given arterial diameter, the AMT of DH group is about $10 \%$ higher than the AMT of TO group, whereas AMT of TO group nearly overlaps that of normal individuals.

\section{Immunohistochemical Analysis}

Immunohistochemical staining for vascular endothelial growth factor (VEGF) was massive and diffuse in all samples of both groups and in normal subjects. Transforming/tumor growth factor (TGF)- $\beta 3$ showed good staining but nonspecific expression sites. There were no differences (i) between $\mathrm{DH}$ and TO groups and (ii) between the experimental groups and normal individuals. Western blot for TGF- $\beta 3$ showed maximum expression in normal individuals, whereas it was decreased in DH group (65\% of that found in normal subjects) and even

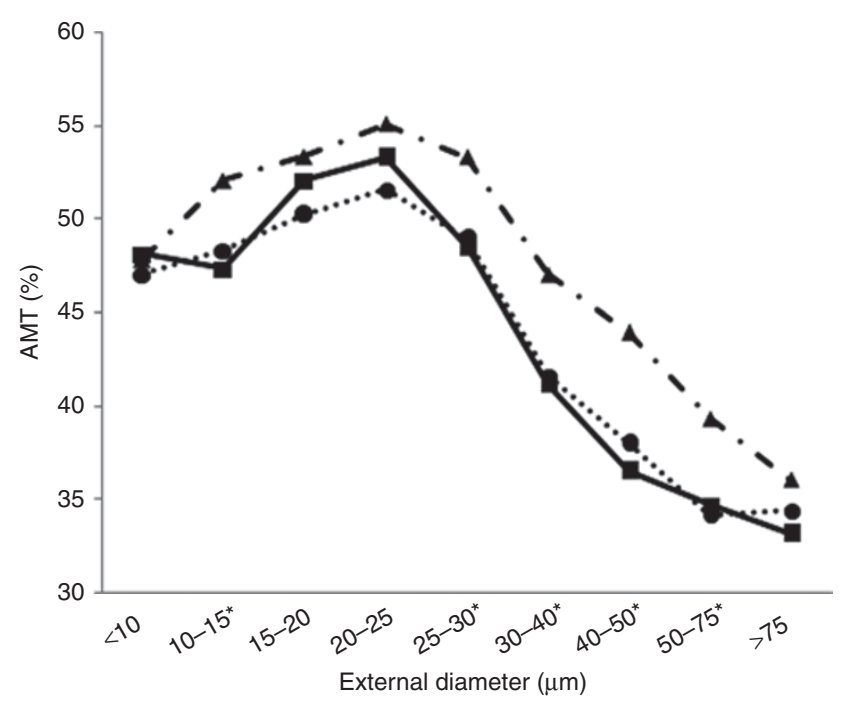

Figure 1 Relationship between arterial size and arterial muscular thickness (AMT). Data on unoperated siblings (N) has been added for normal standard. Squares and continuous line represent the tracheal occlusion (TO) group; triangles and dot-hyphen line show the diaphragmatic hernia (DH) group; the dotted line with circles corresponds to standard $\mathrm{N}$. Arteries have been grouped according to their sizes. The AMT value represents the mean AMT in each range of diameters (shown by a square, a triangle, or a circle according to the groups TO, DH, and N). AMT in DH group is higher than that in TO and $\mathrm{N}$ groups at all arterial sizes. Asterisks show the intervals where there is a significant difference $(P<0.05)$ in the distribution of AMT between DH and TO groups (Mann-Whitney test).

more so in TO group (32\% of that found in normal subjects). Western blot was not available for VEGF.

\section{Variable Correlations}

There is a significant correlation between RAC and PI $(r=$ $-0.528, P=0.043)$. AMT shows a weak correlation with FMBV $(r=-0.379, P=0.133)$. There was no correlation between any other pair of airway/vascular variables: LBWR/PI, LBWR/ FMBV, LBWR/AMT, RAC/FMBV, PI/AMT, and RAC/AMT (Figure 2).

\section{DISCUSSION}

TO has been demonstrated to improve lung hypoplasia associated with $\mathrm{CDH}$ in animal models. Several nonrandomized human studies suggest some benefit of TO for high-risk fetuses (17-19).

We chose the rabbit model because rabbit has a short, multiple, and rather stable gestation at a moderate cost. Its fetuses, however, are large enough to allow hemodynamic examination with a fine-power Doppler ultrasonography machine (16), unlike rat fetuses. This model of $\mathrm{CDH}$ has been validated and repeatedly used (15,20-24).

This study gathered information on living fetuses and combined it with data obtained after killing; this combination provides a unique approach.

The main problems concerning $\mathrm{CDH}$, pulmonary hypoplasia, and persistent pulmonary hypertension are addressed in a simple way: pulmonary hypoplasia is related to development of airway spaces and pulmonary hypertension depends 
a

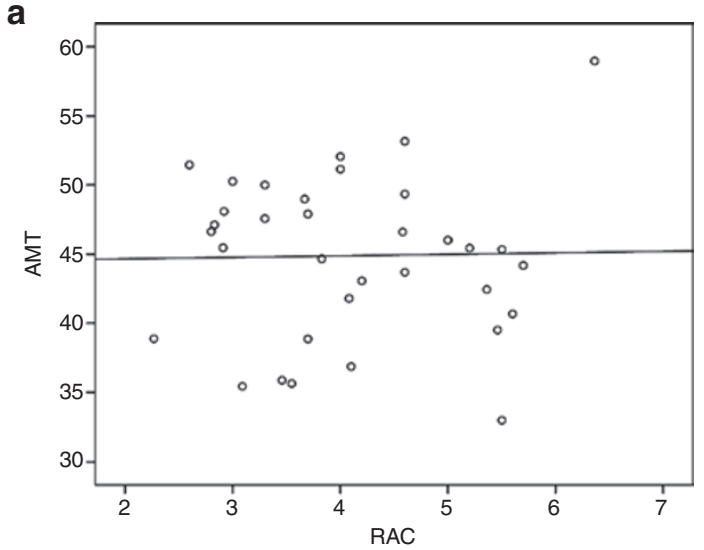

b

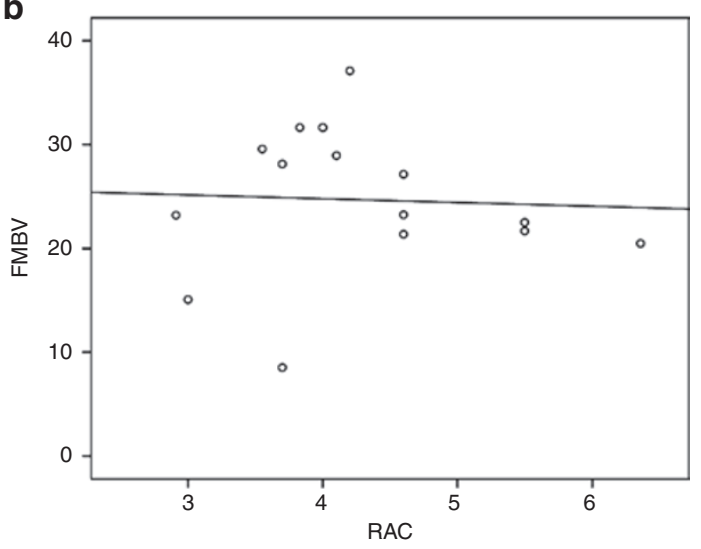

C

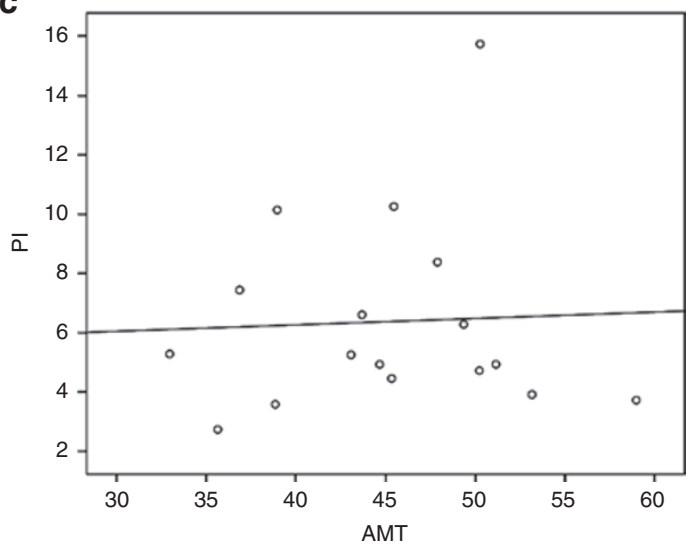

Figure 2 Parameters of pulmonary hypoplasia, arterial thickness, and hemodynamics were compared in all subjects. Correlations show a repeated flat line pattern. The figure provides examples covering the whole possibility of correlations among the three categories of variables: (a) correlation between radial alveolar count (RAC) and arterial muscular thickness (AMT) $\left(R^{2}\right.$ lineal $\left.=6.521 \times 10^{-4}\right)$; (b) correlation between RAC and fractional moving blood volume (FMBV; $R^{2}$ lineal $\left.=0.002\right)$; and (c) correlation between $\mathrm{AMT}$ and pulsatility index $\left(\mathrm{Pl} ; R^{2}\right.$ lineal $\left.=0.002\right)$.

mostly on vascular maturation. TO improves both conditions. Nevertheless, there are no studies that correlate the response to TO observed in airway spaces with reference to the induced vascular maturation. Although both airway and vascular response occur in parallel, it is not known whether one response could predict what will happen to the other. Clinical observations suggest that the correlation between airway development and vascular maturation could be weak. Pathological studies in surviving human newborns are not possible. Only controlled animal studies can provide the lacking knowledge.

Our groups (DH and $\mathrm{TO}$ ) behave basically as expected. Subjects with untreated DH reproduced the main clinical features observed in patients with $\mathrm{CDH}$, namely, herniation of abdominal viscera into the chest, pulmonary hypoplasia, a thick muscular layer in the pulmonary arteries, and poor hemodynamic measurements. In $\mathrm{DH}$ fetuses treated with $\mathrm{TO}$, all these parameters improved significantly. We can thus confirm what has been previously reported on $\mathrm{CDH}$ and fetal therapy with TO: following restriction to lung development caused by DH, TO promotes further growth and maturation, both in terms of airway spaces and vascular tree spread (8).

There are few reports addressing the issue of hemodynamics in $\mathrm{CDH}$ models. PI is a predictor of vascular resistance. In a model for placental blood flow measurements (25), PI decreases as new collateral vessels open, lowering the vascular resistance of the system; and reversely, when small placental arteries are closed, and thus the resistance is high, there is a significant rise in PI. Sylvester et al. (26) reported results similar to ours, using a sheep model: DH was associated with pulmonary hypoplasia and increased PI, which were reversed after TO. These authors also found that fetuses with $\mathrm{DH}$ treated with TO had a more physiological hemodynamic response to maternal hyperoxygenation near term than untreated fetuses with DH. In another study, Moreno-Álvarez et al. (27) correlate the observed-to-expected lung-to-heart ratio with PI and FMBV in human fetuses. They found that the worse the fetal pulmonary hemodynamics, the more hypoplastic are the neonatal lungs. Subsequently, a correlation between hemodynamic parameters and survival rate has been established in human fetuses with CDH $(28,29)$.

The thick muscular layer of pulmonary arteries contributes to increase in the vascular resistance of the pulmonary system. The increase in AMT is one of the main causes of pulmonary hypertension in $\mathrm{CDH}$, other causes being the higher sensitivity of the pulmonary arteries to hypoxia and their relative inability to vasodilate. Figure 1 shows where the maximum vascular resistance takes place. The peak in small arteries $(<30-\mu \mathrm{m}$ diameter) localizes the highest resistance at the intra-acinary level (30-32). There is one other study showing these types of data in a graph, also obtained from the rabbit model (15). In their study, Roubliova et al. did not find a peak in AMT, but a straight descending line from the smaller and thicker arteries to the wider ones with less resistance. Roubliova et al. saw the beneficial effect of TO mainly on small vessels, whereas its effect on larger arteries was less relevant in their work. In this study, the effect of TO in reducing vascular resistance is observed in the whole spectrum of arterial diameters. Moreover, the curve corresponding to the TO group nearly overlaps with that of normal subjects, indicating that $\mathrm{TO}$ tends to restore to normal the muscular thickness of the pulmonary arteries overall. The strength of our data lies on the large amount of arteries measured $(n=1,728)$. 


\section{Articles | Prat Ortells etal.}

The cornerstone of this work is the analysis of the relationship among the different responses to TO. So far, nothing really new has been found; nevertheless, confirmation of previous results is important to validate the model and future conclusions.

Because TO promotes a variety of effects, some degree of correlation is expected among the different responses. For instance, RAC is correlated with PI, and AMT is correlated with FMBV. However, after a detailed examination of all the possible parameter correlations, a flat pattern of no correlation emerged. Although this experiment is based on a small number of cases and it lacks the statistical power to rule out other significant correlations, the flat set of linear correlations is striking. All possible correlations between parameters of pulmonary hypoplasia (RAC and LBWR), hemodynamic measurements (PI and FMBV), and AMT showed this flat pattern, as exemplified in Figure 2.

In human clinical practice, some $\mathrm{CDH}$ fetuses that have been properly treated with TO do not show improvements in their hemodynamic measurements despite showing an apparent growth of their lungs. These fetuses face a bad prognosis compared with those showing normalized hemodynamic status (28). Clinicians are not able to explain why some fetuses respond to the therapy, whereas some others fail. We wonder if the inability to predict the effect of $\mathrm{TO}$ would be explained by a lack of fine correlation between pulmonary growth and pulmonary vascular maturation.

If we assume that there is no correlation among the different responses to TO, we have to infer that TO must trigger different pathways to (i) make the lungs grow and develop the pulmonary airway and respiratory units, (ii) revert the abnormal vascular thickness, and (iii) decrease the overall vascular resistance in a very sensitive arterial system, as estimated by hemodynamic ultrasonography measurements. This idea of multiple pathways would explain the repeated pattern of no correlation and the already mentioned clinical problem.

As a secondary goal, we intended to determine the differential expression of key growth factors regulating critical phases of pulmonary development. Unfortunately, the rabbit model is not the best for immunohistochemistry, due to the lack of antibodies targeted against these factors; most commercial antibodies are made from rabbit serum and are thus not useful in rabbits. It was obvious that anti-VEGF was useless, although the manufacturer does not mention any contraindication for its use in rabbit tissue. The expression of TGF- $\beta 3$, the only growth factor that we have been able to test, is not significantly modified by TO. This suggests that the TGF- $\beta 3$ pathway does not participate in the response to TO. Western blot for TGF- $\beta 3$ was then performed, following a suggestion by our pathologist, believing that this technique could better demonstrate and quantify small differences among groups.

Several weak points of this study can be discussed. Mortality rate was $100 \%$ until we sought advice from one of the researchers publishing on this model $(15,23,33-36)$. After his counseling, we achieved a success rate of $40 \%$ in the first fetal operation, the creation of a DH. Our DH and TO groups were very similar to those previously reported by others $(15,20,21,24)$. Those reports, having a sham group, show enough evidence that there is no effect of surgery itself on lung development. Therefore, we have focused our effort in answering the question whether a relationship exists between airway and vascular lung development after TO. We collected some unoperated littermates just to know where normality is. The number of animals per group (9 and 10) is not small. Nevertheless, as the ultrasonography equipment was not available every harvest day, the hemodynamic evaluation could not be performed in every fetus. The statistical power is therefore not very strong.

The originality of this study is that there was no previous knowledge that airway and vascular development following TO would run independently, their association being weaker than what was expected. There is a need to perform more studies to validate this hypothesis. Simpler models should be designed to test it, such as the murine $\mathrm{CDH}$ model with nitrofen.

In summary, we have measured not just the airway and vascular lung morphometry but also the hemodynamic status in a model of $\mathrm{CDH}$ and TO. This experimental design allows correlation of the well-known histological changes with the hemodynamic status. The main conclusion of this study is that there is no correlation between vascular and airway maturation induced by TO. Therefore, we hypothesize that several molecular pathways regulate the mechanism by which TO achieves its effects.

\section{METHODS}

\section{Study Design}

In this experimental study, two groups of animals were compared: $\mathrm{DH}$ (a rabbit model of $\mathrm{CDH}$ ) and TO (DH animals treated later with $\mathrm{TO}$ ). Just before birth, the hemodynamic parameters of the pulmonary circulation were measured in vivo. Autopsy was conducted after birth, and data on pulmonary development, both of airspaces and vascular tree, were collected. Comparison and adequate statistical analysis was done between groups, especially between pairs of variables (one airway and one vascular variable, in all combinations).

\section{Variables}

To estimate airway development, LBWR and RAC were measured. Both parameters are quantitative estimators of pulmonary hypoplasia. RAC is even more sensitive than LBWR, and it provides an idea of airway maturation. Vascular response was analyzed in two ways: a functional, hemodynamic measurement; and a structural, histological study. In a previous study (16), our group found that PI and FMBV specifically characterize the hemodynamic response to TO. Consequently, for this study, we recorded PI and FMBV at the time of delivery. To have a quantitative parameter of pulmonary vascular development, AMT was chosen. AMT is known to be an estimator of vascular maturity because thickening of the arterial muscular layer is the most striking change in the vascular appearance of $\mathrm{CDH}$ fetuses.

\section{The Rabbit Model of CDH}

We used the rabbit model of DH and TO published by Roubliova et al. $(15,24)$. Two experimental groups were defined: the DH group consisted of newborn rabbits with surgically created $\mathrm{DH}$ on gestational day (GD) 23; the TO group consisted of newborn rabbits with DHas in $\mathrm{DH}$ group -in which the trachea had been surgically ligated on GD 28. All animals were collected at GD 30, along with one unoperated sibling per litter.

\section{Surgical Protocol}

The Animal Ethical Committee (Comitè Ètic d'Experimentació Animal, Universitat de Barcelona, Spain) approved the following 
protocol. Pregnant New Zealand White does were brought to our facility on GD 19 from a specialized farm (Granja Cunícola San Bernardo S.L., carretera de Tarazona s/n, Tulebras, Navarra, Spain). On GD 23, the first surgical procedure was carried out under sterile conditions. Anesthesia was induced with ketamine $(20 \mathrm{mg} / \mathrm{kg})$ and chlorpromazine $(2.5 \mathrm{mg} / \mathrm{kg})$ and maintained with inhalation of isoflurane $(2-3.5 \%)$ in $\mathrm{O}_{2}(1 \mathrm{l} / \mathrm{min})$, administered through a facial mask. A $20 \mathrm{ml} / \mathrm{kg}$ bolus of normal saline solution was intravenously administered throughout the procedure. Antibiotic prophylaxis with cefazolin $(50 \mathrm{mg} / \mathrm{kg})$, meloxicam $(1 \mathrm{mg} / \mathrm{kg})$, and depoprogesterone (6 $\mu \mathrm{g} / \mathrm{kg}$ ) - as uterine inhibitor-were given intramuscularly. Boluses of intravenous fentanyl were given as needed during the procedure. A midline laparotomy exposed the two uterine horns. Ultrasonographic data were obtained from the two lateral fetuses and another random one, directly placing the probe on the uterus. A hysterotomy on the cephalic pole allowed exposure of the fetal head, thorax, and upper abdomen. The diaphragm was exposed through a low left thoracotomy, incised, and widely opened. The left lobe of the liver herniated into the chest. The thoracotomy was closed in a single layer with one or two nonabsorbable 6-0 sutures. The fetus was then replaced into the amniotic cavity, which was closed together with the uterus with a running 4-0 silk suture; amniotic fluid was restored with warm saline to a pressure similar to that of the adjacent amniotic sacs. Laparotomy was closed in two layers, isoflurane was discontinued, and the doe recovered. A second dose of meloxicam was administered $12 \mathrm{~h}$ after the procedure.

On GD 28, a second fetal operation was performed following the same protocol. Ultrasound data were obtained from all survivors and one healthy littermate. The fetal head was exposed through a new hysterotomy; a midline cervicotomy allowed isolation of the trachea from the esophagus. The trachea was ligated with a 3-0 silk; the fetus was replaced, leaving the neck unsutured, and the hysterotomy was closed as described. A third laparotomy was done on GD 30 (term = $31 \mathrm{~d}$ ), and hemodynamic data were obtained from all operated survivors and one control littermate, immediately before harvesting the fetuses through a Cesarean delivery.

\section{Hemodynamics}

A high-resolution ultrasound machine (Siemens Sonoline Antares, by Siemens Medical Systems, Malvern, PA) equipped with a $13-\mathrm{MHz}$ linear transducer was used to register the following hemodynamic data: PI, peak of early diastolic reversed flow, peak of maximum systolic speed, and FMBV. Recordings were taken of the right pulmonary artery in its intrapulmonary portion-the left one being too small to allow any Doppler exam-seen at the "four chambers view" of the heart. At least five recordings per subject were conducted, at different phases of the cardiac cycle, including no less than three consecutive beats free of the interference of fetal movements. For this study, we only considered PI and FMBV on GD 30; the early measurements have already been published (16). Measurements on GD 30 were taken directly on the fetus, which was harvested immediately afterward; therefore, a full follow-up of every subject is warranted.

\section{Sample Processing}

The fetuses were weighed, killed, and autopsied. DH was confirmed. The lungs were removed, weighed, labeled, and snap-frozen in liquid nitrogen for conservation at $-70^{\circ} \mathrm{C}$ or fixed in buffered formalin. LBWR was calculated according to the formula: LBWR $=$ (left lung weight + right lung weight)/body weight $\times 100$.

\section{Histological Study}

A coronal section of every left lung, including both lobes and the hilum, was examined after hematoxylin and eosin staining. From every lung section, the following were determined:

Radial alveolar count. This is the number of alveoli crossed by the shortest line running from a respiratory bronchiole to the edge of the acinus. Ten counts were taken of every lung, following the original description of the method by Emery and Mithal (37). This is a fine histological parameter of pulmonary development, relating exponentially to the number of airspaces filling from one respiratory bronchiole.
Arterial muscular thickness. The internal diameter (ID) and the external diameter (ED) of the intrapulmonary arterial branches were recorded. The proportional muscular arterial thickness was calculated according to the formula AMT $=(E D-I D) / E D \times 100$. At least 50 arteries were measured from each left lung. To reduce bias cut error, arteries in which the section was not round were rejected if the longest diameter was greater than twice the shortest diameter.

\section{Immunohistochemical Analysis}

Standard immunohistochemical analysis was used to determine expression of VEGF and TGF- $\beta 3$. These are the only growth factors related to pulmonary development that have commercially available antibodies against rabbit tissue samples: monoclonal anti-VEGF ab46154 (Abcam, Cambridge, MA) and monoclonal anti-TGF- $\beta 3$, Clone Xn-12 (Santa Cruz Biotechnology, Santa Cruz, CA). Western blot was done when available, in case of unsuccessful immunohistochemical technique. A standard western blot technique was performed, using sheep anti-mouse horseradish-peroxidase-conjugated antibody (Amersham, Aylesbury, UK) as a second antibody. Western blot images were acquired with Fuji Film Image Reader LAS 3000 and quantified with Image J software (Rasband WS. ImageJ, US National Institutes of Health, Bethesda, MD).

\section{Statistical Analysis}

Variables were compared between groups. Quantitative variables were treated with the appropriate statistical tests, using the Statistical Package for the Social Sciences (SPSS 20.0; SPSS, Chicago, IL). Statistical significance was established at $P \leq 0.05$. Variables were also paired (one airway-related variable with one vasculature-related variable), and regression lines were drawn for correlations.

\section{ACKNOWLEDGMENTS}

The authors thank Jacques Jani for his invaluable and kind help with mastering this demanding animal model.

\section{STATEMENT OF FINANCIAL SUPPORT}

This work was supported by the Fondo de Investigación Sanitaria (FIS PI 06/0865; Spain). J.P.O. had a Scholarship from Fundació Sant Joan de Déu (Barcelona, Spain).

Disclosure: The authors declare no relevant or material financial interest that relate to the research described in this article.

\section{REFERENCES}

1. Keller RL. Antenatal and postnatal lung and vascular anatomic and functional studies in congenital diaphragmatic hernia: implications for clinical management. Am J Med Genet C Semin Med Genet 2007;145C: 184-200.

2. Thébaud B, Mercier JC, Dinh-Xuan AT. Congenital diaphragmatic hernia. A cause of persistent pulmonary hypertension of the newborn which lacks an effective therapy. Biol Neonate 1998;74:323-36.

3. Beals DA, Schloo BL, Vacanti JP, Reid LM, Wilson JM. Pulmonary growth and remodeling in infants with high-risk congenital diaphragmatic hernia. J Pediatr Surg 1992;27:997-1001; discussion 1001-2.

4. Keijzer R, Puri P. Congenital diaphragmatic hernia. Semin Pediatr Surg 2010;19:180-5.

5. de Buys Roessingh AS, Dinh-Xuan AT. Congenital diaphragmatic hernia: current status and review of the literature. Eur J Pediatr 2009;168:393406.

6. Mohseni-Bod H, Bohn D. Pulmonary hypertension in congenital diaphragmatic hernia. Semin Pediatr Surg 2007;16:126-33.

7. DiFiore JW, Fauza DO, Slavin R, Peters CA, Fackler JC, Wilson JM. Experimental fetal tracheal ligation reverses the structural and physiological effects of pulmonary hypoplasia in congenital diaphragmatic hernia. J Pediatr Surg 1994;29:248-56; discussion 256-7.

8. Khan PA, Cloutier M, Piedboeuf B. Tracheal occlusion: a review of obstructing fetal lungs to make them grow and mature. Am J Med Genet C Semin Med Genet 2007;145C:125-38.

9. Grushka JR, Al-Abbad S, Baird R, Puligandla P, Kaplan F, Laberge JM. The effect of in vitro tracheal occlusion on branching morphogenesis in fetal 
lung explants from the rat nitrofen model of congenital diaphragmatic hernia. J Pediatr Surg 2010;45:943-7.

10. DiFiore JW, Fauza DO, Slavin R, Wilson JM. Experimental fetal tracheal ligation and congenital diaphragmatic hernia: a pulmonary vascular morphometric analysis. J Pediatr Surg 1995;30:917-23; discussion 923-4.

11. Benachi A, Chailley-Heu B, Delezoide AL, et al. Lung growth and maturation after tracheal occlusion in diaphragmatic hernia. Am J Respir Crit Care Med 1998;157(3 Pt 1):921-7.

12. Kitano Y, Davies P, von Allmen D, Adzick NS, Flake AW. Fetal tracheal occlusion in the rat model of nitrofen-induced congenital diaphragmatic hernia. J Appl Physiol 1999;87:769-75.

13. Kanai M, Kitano Y, von Allmen D, Davies P, Adzick NS, Flake AW. Fetal tracheal occlusion in the rat model of nitrofen-induced congenital diaphragmatic hernia: tracheal occlusion reverses the arterial structural abnormality. J Pediatr Surg 2001;36:839-45.

14. Chapin CJ, Ertsey R, Yoshizawa J, et al. Congenital diaphragmatic hernia, tracheal occlusion, thyroid transcription factor-1, and fetal pulmonary epithelial maturation. Am J Physiol Lung Cell Mol Physiol 2005;289:L44-52.

15. Roubliova XI, Verbeken EK, Wu J, Vaast P, Jani J, Deprest JA. Effect of tracheal occlusion on peripheric pulmonary vessel muscularization in a fetal rabbit model for congenital diaphragmatic hernia. Am J Obstet Gynecol 2004;191:830-6.

16. Cruz-Martinez R, Moreno-Alvarez O, Prat J, et al. Lung tissue blood perfusion changes induced by in utero tracheal occlusion in a rabbit model of congenital diaphragmatic hernia. Fetal Diagn Ther 2009;26:137-42.

17. Deprest J. Towards an endoscopic intra-uterine treatment for congenital diaphragmatic hernia. Verh K Acad Geneeskd Belg 2002;64:55-70.

18. Deprest JA, Nicolaides K, Gratacos E. Fetal surgery for congenital diaphragmatic hernia is back from never gone. Fetal Diagn Ther 2011;29:617.

19. Jani JC, Nicolaides KH, Gratacós E, et al. Severe diaphragmatic hernia treated by fetal endoscopic tracheal occlusion. Ultrasound Obstet Gynecol 2009;34:304-10.

20. Wu J, Yamamoto H, Gratacos E, et al. Lung development following diaphragmatic hernia in the fetal rabbit. Hum Reprod 2000;15:2483-8.

21. Wu J, Ge X, Verbeken EK, Gratacós E, Yesildaglar N, Deprest JA. Pulmonary effects of in utero tracheal occlusion are dependent on gestational age in a rabbit model of diaphragmatic hernia. J Pediatr Surg 2002;37:11-7.

22. Fauza DO, Tannuri U, Ayoub AA, Capelozzi VL, Saldiva PH, Maksoud JG. Surgically produced congenital diaphragmatic hernia in fetal rabbits. J Pediatr Surg 1994;29:882-6.

23. Roubliova XI, Lewi PJ, Verbeken EK, et al. The effect of maternal betamethasone and fetal tracheal occlusion on pulmonary vascular morphometry in fetal rabbits with surgically induced diaphragmatic hernia: a placebo controlled morphologic study. Prenat Diagn 2009;29:674-81.
24. Roubliova X, Verbeken E, Wu J, et al. Pulmonary vascular morphology in a fetal rabbit model for congenital diaphragmatic hernia. J Pediatr Surg 2004;39:1066-72.

25. Thompson RS, Trudinger BJ. Doppler waveform pulsatility index and resistance, pressure and flow in the umbilical placental circulation: an investigation using a mathematical model. Ultrasound Med Biol 1990;16:449-58.

26. Sylvester KG, Rasanen J, Kitano Y, Flake AW, Crombleholme TM, Adzick NS. Tracheal occlusion reverses the high impedance to flow in the fetal pulmonary circulation and normalizes its physiological response to oxygen at full term. J Pediatr Surg 1998;33:1071-4; discussion 1074-5.

27. Moreno-Alvarez O, Cruz-Martinez R, Hernandez-Andrade E, et al. Lung tissue perfusion in congenital diaphragmatic hernia and association with the lung-to-head ratio and intrapulmonary artery pulsed Doppler. Ultrasound Obstet Gynecol 2010;35:578-82.

28. Cruz-Martinez R, Moreno-Alvarez O, Hernandez-Andrade E, et al. Contribution of intrapulmonary artery Doppler to improve prediction of survival in fetuses with congenital diaphragmatic hernia treated with fetal endoscopic tracheal occlusion. Ultrasound Obstet Gynecol 2010;35:572-7.

29. Moreno-Alvarez O, Hernandez-Andrade E, Oros D, Jani J, Deprest J, Gratacos E. Association between intrapulmonary arterial Doppler parameters and degree of lung growth as measured by lung-to-head ratio in fetuses with congenital diaphragmatic hernia. Ultrasound Obstet Gynecol 2008;31:164-70.

30. Kay JM. Comparative morphologic features of the pulmonary vasculature in mammals. Am Rev Respir Dis 1983;128(2 Pt 2):S53-7.

31. Geggel RL, Murphy JD, Langleben D, Crone RK, Vacanti JP, Reid LM. Congenital diaphragmatic hernia: arterial structural changes and persistent pulmonary hypertension after surgical repair. J Pediatr 1985;107:45764.

32. Laudy JA, Wladimiroff JW. The fetal lung. 1: developmental aspects. Ultrasound Obstet Gynecol 2000;16:284-90.

33. Jani JC, Flemmer AW, Bergmann F, et al. The effect of fetal tracheal occlusion on lung tissue mechanics and tissue composition. Pediatr Pulmonol 2009;44:112-21.

34. Gallot D, Coste K, Jani J, et al. Effects of maternal retinoic acid administration in a congenital diaphragmatic hernia rabbit model. Pediatr Pulmonol 2008;43:594-603.

35. Flemmer AW, Jani JC, Bergmann F, et al. Lung tissue mechanics predict lung hypoplasia in a rabbit model for congenital diaphragmatic hernia. Pediatr Pulmonol 2007;42:505-12.

36. Coste K, Gallot D, Marceau G, et al. Expression of retinoid receptors during rabbit lung development. Animal 2007;1:403-9.

37. Emery JL, Mithal A. The number of alveoli in the terminal respiratory unit of man during late intrauterine life and childhood. Arch Dis Child 1960;35:544-7. 